\title{
Enhancement of Voltage Stability by optimal location of UPFC using MPSO and Power Flow Analysis using ECI Algorithm
}

\author{
${ }^{1}$ Mr. P Harshavardhan Reddy, ${ }^{2}$ Dr. M Padma Lalitha, ${ }^{3}$ Mr. Kjv Satish Babu \\ ${ }^{I}$ Assistant professor, EEE Department, Annamacharya institute of Technology and sciences, Rajampeta \\ ${ }^{2}$ Professor \& HOD, EEE Department, Annamacharya institute of Technology and sciences, Rajampeta \\ ${ }^{3} P G$ Student, EEE Department, Annamacharya institute of Technology and sciences, Rajampeta
}

\begin{abstract}
Voltage instability and voltage collapse have been considered as a major threat to present power system networks due to their stressed operation. It is very important to do the power system analysis with respect to voltage stability. Flexible AC Transmission System (FACTS) is an alternating current transmission system incorporating power electronic-based and other static controllers to enhance controllability and increase power transfer capability. A FACTS device in a power system improves the voltage stability, reduces the power loss and also improves the load ability of the system. FACTS have made the power systems operation more flexible and secure. Amongst the several FACTS controllers, the Unified Power Flow Controller (UPFC) is most effective to improve the enhancement of voltage stability and reduces the power loss. This study investigates the application of Particle Swarm Optimization (PSO) to find sizing of Unified Power Flow Controller (UPFC) device to minimize the voltage stability index, total power loss, load voltage deviation, and cost of FACTS devices to improve voltage stability in the power system. A new model is proposed in this thesis to improve existing power-based model by using the Norton Equivalent Theorem. The proposed model can be integrated with the Equivalent Current Injection (ECI) power flow model easily. By ECI algorithm, it is much quickly and precisely to implement power flow calculations. Finally It is observed from the results that the voltage stability margin is improved, the voltage profile of the power system is increased and real power losses also reduced by optimally sizing UPFC device in the power system.
\end{abstract}

Index Terms: Voltage stability, Unified Power Flow Controller (UPFC), Equivalent-Current Injection (ECI), Modified particle swarm optimization (MPSO)

\section{Introduction}

Power system networks are complex systems that are nonlinear, non-stationary, and prone to disturbances and faults. Reinforcement of a power system can be accomplished by improving the voltage profile, increasing the transmission capacity and others.

Flexible AC Transmission System (FACTS) devices are an alternate solution to address some of those problem. The FACTS devices can be categorized into three types, such as series controllers, shunt controllers and combined series-shunt controllers. In principle, the series controllers inject voltage in series with the line and the shunt controllers inject current into the system at the point of connection. The combined series-shunt controllers inject current into the system with the shunt part of the controllers and voltage in series in the line with the series part of the controllers. In the case of voltage support, shunt FACTS devices, such as STATCOM and SVC are typically used. This study is focused on the steady state performance of multiple UPFC devices in the power system. Particularly, it is desired to determine their optimal location and capacity.

In order to overcome these problems, Evolutionary Computation Techniques have been employed to solve the optimal allocation of FACTS devices. Traditional optimization methods such as mixed integer linear and non linear programming have been investigated to address this issue. This paper applied the ability of the modified particle swarm optimization (MPSO) efficiency. The objective of MPSO is to improve the searching quality of ants by optimizing themselves to generate a better result. This method can not only enhance the neighborhood search, but can also search the optimum solution quickly to advance convergence.

The load flow analysis (commonly called load flow or power flow) is the basic tool for investigating power system state variables, and it is very important part of the system supervisory, planning and optimal operation. The unbalance three-phase load flows based on the Equivalent-Current-Inject (ECI) were applied successfully to the distribution system. It is unable to apply the ECI model to the high voltage transmission systems, because of the voltage - controlled buses (PV Bus). In this a new power flow approach based on ECI model and Cartesian coordination is presented. PV Bus model were developed, and according to the network characteristics, the decoupled models were also proposed.

This paper introduces the application of MPSO for optimal location and capacity of a new UPFC with ECI model in the power system. It is organized as follows: Section IIUPFC with ECI. Section III presents the basic concepts of modified particle swarm optimization (MPSO). In section IV the objective function to be 
optimized is described. In section V simulation results are presented. In section VI conclusions and future work are given.

\section{A. UPFC Basic Concept}

\section{Upfc With Eci Model}

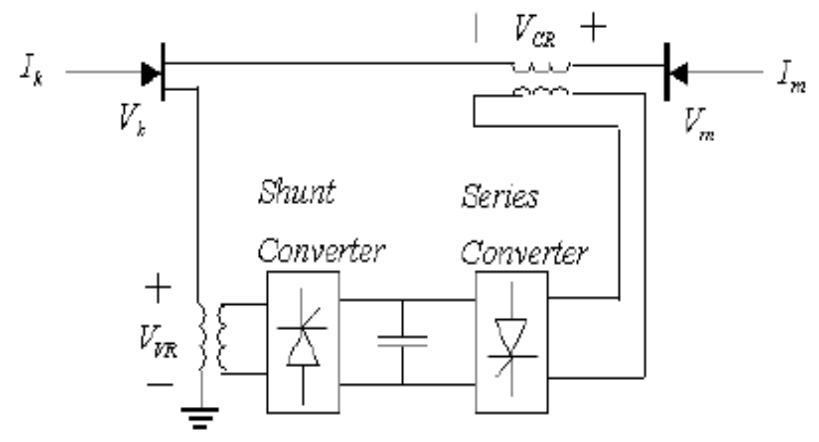

Fig. 1 UPFC connected to power system

The Fig. 1 shows a conceptual representation of UPFC in a two-machine power system. The series branch of UPFC is modeled as a generalized synchronous voltage, and represented at the power system frequency by voltage phasor $V_{C R}$ and its phase angle $\rho$ in series with the transmission line. Therefore, it is clear that the effective sending end voltage is modified by the UPFC series injected voltage in both magnitude and it phase angle, and as a result it is able to control, by adjusting the magnitude and the phase of $V_{C R}$, the transmittable active power as well as the reactive power. Moreover, in dynamic control applications, it is also able to provide power oscillation damping by real-time modulating the real power of the ac system. This is the result of its ability to alternatively insert a virtual positive and negative damping resistor in series with the line in accordance with the angular acceleration and deceleration of the disturbed generators. The UPFC consists of two voltage sourced converters one is Series Converter and other is Shunt Converter and are designed to be operated from a common DC link voltage supported by a DC storage capacitor.

In Series Converter, the series branch, operated as a SSSC, is used to perform the main control functions of a UPFC. It generates voltage, $V_{C R}$, at the system frequency controlled by a proper switching control technique. During the operation the voltage, $V_{C R}$, is added to the AC system terminal voltage $V_{k}$ by the series connected injection series transformer, $T_{s e}$. The transmission line current flows through this voltage source resulting in reactive and active power exchange between it and the ac system. The reactive power exchanged at the ac terminal is generated internally by the converter. The active power exchanged at the ac terminal is converted into dc power which appears at the dc link as a positive or negative active power demand. In normal operations, the phase angle of the series voltage can be chosen independently of the line current between 0 and $2 \pi$, and its magnitude can be varied between zero and a pre-specified maximum value. Therefore the real power can freely flow in either direction between the AC terminals of the two converters and each converter can also generate or absorb reactive power independently at its own AC output terminals to affect system voltages.

Shunt Converter connected in shunt with the AC power system via a shunt transformer $T_{s h}$ operated as a STATCOM, is used primarily to provide the real power demand of series converter at the common DC link terminal from the AC power system. Since shunt converter can also generate or absorb reactive power at its AC terminal, independently of the real power transferred to (or from) the DC terminal. It follows that, with proper controls, it can also fulfill the function of an independent STATCOM operations providing reactive power compensation for the transmission line and thus executing an indirect voltage regulation at the input terminal of the UPFC. It is important to note that there is a closed direct path for the active power negotiated by the action of series voltage injection through two converters back to the line, the corresponding reactive power exchanged is supplied or absorbed locally by series converter and therefore doesn't have to be transmitted by the line. Thus, shunt converter can be operated at a unity power factor or controlled to have a reactive power exchange with the line independent of the reactive power exchanged by converter 2. Obviously, there is no reactive power flow through the UPFC dc link. In addition, the UPFC has the flexibility the control either its series or shunt branch or both to achieve a desired effect on the power flow transmitted between two buses.

The UPFC active power $P_{m}$ and reactive power $Q_{m}$ is,

$$
\begin{gathered}
P_{m}=\frac{\left(V_{k}+V_{C R}\right) \cdot V_{m}}{X} \sin \delta \\
Q_{m}=\frac{\left(V_{k}+V_{C R}\right) \cdot V_{R}}{X}(1-\cos \delta)
\end{gathered}
$$


Where,

$$
\begin{aligned}
& \mathrm{X}=\text { coupling transformer equivalent Reactance } \\
& \delta=\theta_{\mathrm{k}}-\theta_{\mathrm{m}}
\end{aligned}
$$

In voltage control mode, the reactive current is automatically regulated to maintain the transmission line voltage at the point of connection to a reference value, with a defined droop characteristic. The droop factor defines the per unit voltage error per unit of reactive current within the current range of the converter. The convert supplies leading current to the AC system if the converter output voltage $V_{s h}$ is made to lead the corresponding AC system voltage $V_{k}$. Then it supplies reactive power to the AC system by capacitive operation. Conversely, the converter absorbs lagging current from the AC system; if the converter output voltage $V_{s h}$ is made to lag the AC system voltage $V_{k}$ then it absorbs reactive power to the AC system by inductive operation. If the output voltage is equal to the $\mathrm{AC}$ system voltage, the reactive power exchanges.

\section{B. UPFC with ECI}

The UPFC can act as on equivalent voltage source series reactance. Voltage source can transform the current source by way of Norton Theorem of the $\pi$-circuit as shown in Fig 2 . It is important to note that there is a closed direct path for the active power negotiated by the action of series voltage injection through converter 1 and 2 back to the line.

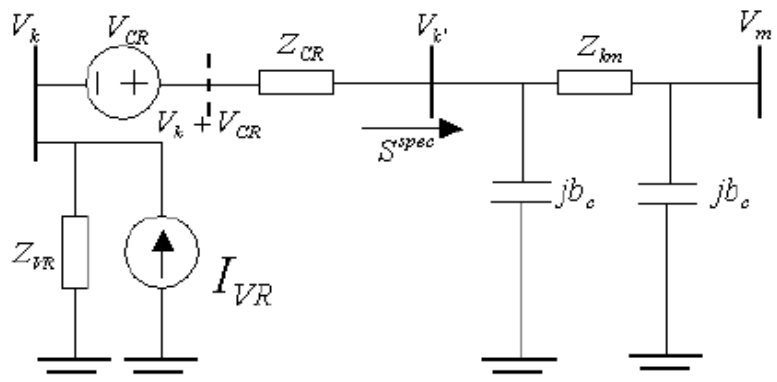

Fig. 2 Transmission line equivalent model UPFC equivalent circuit diagram

According to Fig 2 with ECI model inferential reasoning as follows in equations.

$$
I_{V R}=V_{V R} / Z_{V R}
$$

That is the device does not generate or absorb active power internally. This constraint can be stated as,

$$
\begin{aligned}
P_{C R} & =P_{V R} \\
S_{V R} & =V_{k} I^{*} \\
& =V_{k}\left(\frac{V_{k}-V_{V R}}{z_{V r}}\right) \\
& =\frac{\|\left. v_{2}\right|^{2}}{z_{V R}^{*}} \cdot V_{k}\left(\frac{V_{V R}}{z_{V R}}\right) \\
& =\frac{\left|V_{k}\right|^{2}}{z_{V R}^{*}}-V_{k} I_{V R}^{*}
\end{aligned}
$$

where, $\quad I_{V R}=$ shunt branch current

$V_{V R}=$ shunt branch voltage

$Z_{V R}=$ shunt branch impedance equals

$$
R_{V R}+j X_{V R}
$$

$P_{C R}, P_{V R}=$ the active powers supplied or absorbed

in the series and shunt converters

According to the Newton-Raphson algorithm [4], the ECI mismatch equation with UPFC model can be written a new admittance matrix as,

$$
\begin{aligned}
& I_{k, k}=S^{\text {spec }} / V_{k}, \\
& I_{k, k}=\left(V_{k}+V_{C R}-V_{K},\right)\left(g_{c r}+j b_{c r}\right) \\
& I=Y_{\text {maty }}^{\text {mel }} V
\end{aligned}
$$

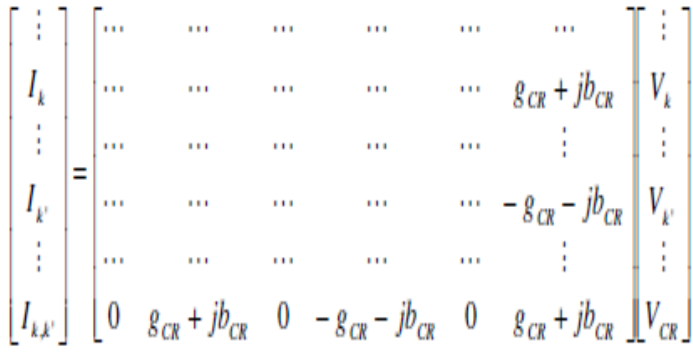


Where, $g_{C R}+j b_{C R}=1 /\left(Z_{C R}\right), S^{\text {spec }}$ is the specified constant apparent power.

$$
Y_{G}^{\text {new }}=\operatorname{Re}\left(Y_{\text {matrix }}^{\text {new }}\right), Y_{B}^{\text {new }}=\operatorname{Im}\left(Y_{\text {matrix }}^{\text {new }}\right)
$$

\section{Modified Particle Swarm Optimization}

\section{A. Basic PSO}

PSO is an evolutionary computation technique developed by Eberhart and Kennedy in 1995, and was inspired by the social behavior of bird flocking and fish schooling. PSO has its roots in artificial life and social psychology as well as in engineering and computer science. It utilizes a population of individuals, called particles, which fly through the problem hyperspace with some given initial velocities. In each iteration, the velocities of the particles are stochastically adjusted considering the historical best position of the particles and their neighborhood best position; where these positions are determined according to some predefined fitness function. Then, the movement of each particle naturally evolves to an optimal or near-optimal solution. The name of "swarm" comes from the irregular movements of the particles in the problem space, more similar to a swarm of mosquitoes rather than flock of birds or school of fish.

At each iteration, the velocity of a particle is determined by both the individual and group experience,

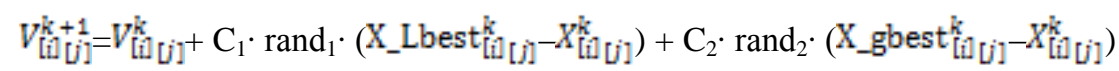

$X_{[\text {[I }[j]}^{k+1}=X_{\text {[i] }[j]}^{k}+V_{[i][1]}^{k+1}$

Where, $[\mathrm{i}],[\mathrm{j}]=$ Population number and particles $V_{\text {[i1 }}^{k}=$ Velocity of the particle in the $\mathrm{k}_{\mathrm{th}}$ $X_{[1][1]}^{k}=$ Position of the particle in the $\mathrm{k}_{\mathrm{th}}$ $\mathrm{X}_{-}$Lbest $\mathrm{t}_{[\mathrm{in}}^{\mathrm{k}}[\mathrm{j}]=\mathrm{I}_{\mathrm{th}}$ fitness best in the $\mathrm{k}_{\mathrm{th}}$ $\mathrm{X}_{\_} \mathrm{g}$ best $\mathrm{Ti1}_{[\mathrm{i} 1}^{\mathrm{k}}=$ Population global best in the

$C 1, C 2=$ Cognitive and Social component: personal best and the global best rand $_{1}$, rand $_{2}=$ Uniform random numbers between 0 (respectively)

The maximum allowable velocity for the particles is controlled by the parameter $V_{\max }$. If $V_{\max }$ is too high, then particles tend to move beyond a good solution; on the other hand, if $V_{\max }$ is small, then particles can be trapped in local minima.

\section{B. Modified PSO}

A weight factor, $\mathrm{W}_{k}$, was added to the previous velocity of the particle. This allows control on the mechanism responsible for the velocities magnitude, which fosters the danger of swarm explosion and divergence, or fast convergence and being trapped in local minima.

Thus, equation (10) can be re-written including the weight factor $\mathrm{W}_{k}$

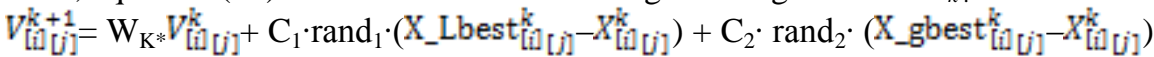

$$
\begin{aligned}
& X_{[\mathrm{i}[j]}^{k+1}=X_{[i[j]}^{k}+V_{[i][j]}^{k+1}
\end{aligned}
$$

The second challenge is to find a feasible weight factor that prevents prematurely because it affects the convergence and the ability of the swarm to find the optimum. A suitable value of $\mathrm{W}_{k}$ provides the desired balance between the global and local exploration ability of the swarm and, consequently, improves the effectiveness of the algorithm. At the beginning, a large inertial weight is better because it gives priority to global exploration of the search space. It can be gradually decreased so as to obtain refined solutions. To introduce chaotic behavior, the iterator called Logistic Map is defined by the following equation,

$$
f_{k}=\mu \cdot f_{k-1 \cdot}\left(1-f_{k-l}\right)
$$

Where $\mu$ is a control parameter and has a real value between 0 and 4 . Despite the apparent simplicity of the equation, the solution exhibits a rich variety of behaviors. The value of $\mu$ determines whether $f_{k}$ stabilizes at a constant size, oscillates between a limited sequence of sizes, or behaves chaotically in an unpredictable pattern. And also the behavior of the system is sensitive to initial values of $f_{k}$. Equation (12) displays chaotic dynamics when $\mu=4.0$ and $f_{0} \epsilon$ $\{0,0.25,0.5,0.75,1.0\}$ [18]. after some tests, the value chosen for $\mathrm{W}_{0}, \mu$ and $f_{0}$ are $3.5,4.0$ and 0.65 , respectively. Therefore, the weight inertial factor is,

$$
\omega_{\mathrm{k}}=\left\{\frac{\varpi_{0}}{1+(\log \mathrm{k})^{2}}\right\} \cdot\left(\mathrm{f}_{\mathrm{k}}\right)
$$




\section{Objective Function}

In such a power network, it is desirable to keep the voltage deviations between $\pm 5 \%$ to avoid voltage collapses during faulty conditions. In general, if the load requirements increase, the voltages at the corresponding buses may drop below 0.95p.u. and consequently an additional voltage support is needed at that particular bus. In this study, the voltage support will be provided by a UPFC with ECI model, and its optimal location and capacity will be determined by using MPSO.

For instance, the IEEE 30-Bus system in Fig.3 has 5generators buses where voltage is regulated by the generator AVRs. These generator buses do not need a UPFC and are omitted from the MPSO search process. Also considering the topology of the system, the bus numbers are limited to the range from 1 to 30 .

\section{UPFC selection to install in a location}

1. Because UPFC are expensive, therefore the minimum device installed is searched for economic efficiency reasons.

2. Generator buses where voltages are regulated by the generator do not need UPFC installation.

3. Each bus is limited to the installation of one device. Installing more does not represent a significant effect.

4. If the bus voltage is above 0.95p.u then UPFC is not installed.

\section{Results}

A 30-Bus test system as shown in Fig. 3 is used for this paper. The test system consists of 5 generators and $24 \mathrm{PQ}$ bus (or load bus). The problem to be addressed consists of finding the optimal location (bus number) and power rating (MVA) of UPFC with ECI model. In this case the MPSO is able to find different options for capacity of the UPFC with the ECI model.

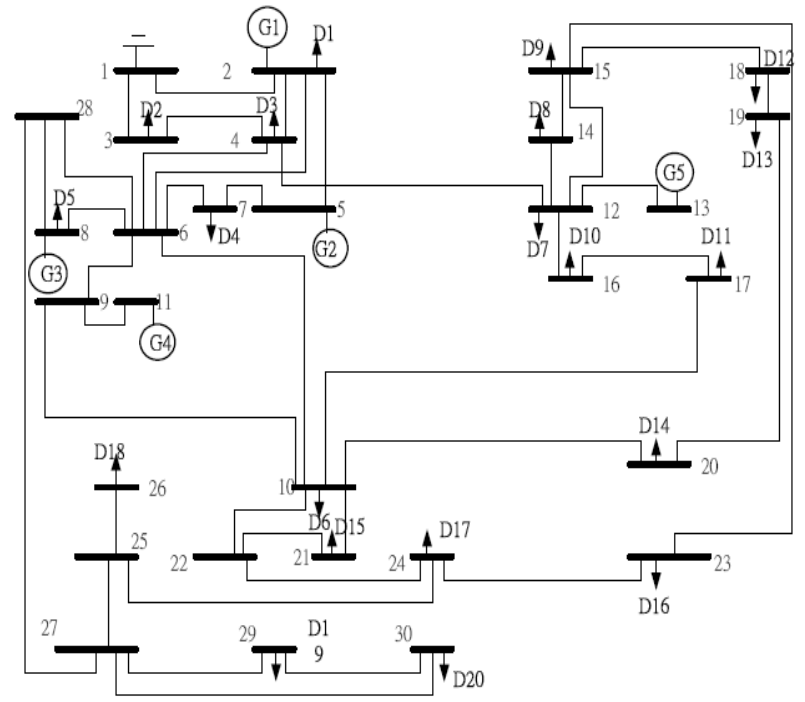

Fig. 3 The IEEE 30-bus test system

\section{WITHOUT UPFC:}

TABLE 1

BUS VOLTAGE AND POWER FLOW RESULT WITHOUT UPFC

\begin{tabular}{|c|c|c|c|c|}
\hline $\begin{array}{c}\text { BUS } \\
\text { No. }\end{array}$ & $\begin{array}{c}\mathbf{V}_{\text {mag }} \\
(\mathbf{p u})\end{array}$ & $\begin{array}{c}\text { Angle } \\
(\mathbf{d e g r e e})\end{array}$ & $\begin{array}{c}\text { P Flow } \\
(\mathbf{p u})\end{array}$ & Q Flow (pu) \\
\hline 1 & 0.8480 & 0 & 2.0876 & -16.3343 \\
\hline 2 & 0.8360 & -4.3026 & 0.1464 & 34.6956 \\
\hline 3 & 0.8169 & -6.0229 & -0.0192 & -0.9600 \\
\hline 4 & 0.8098 & -7.4235 & -0.0608 & -1.2800 \\
\hline 5 & 0.8080 & -11.3190 & -0.7536 & 13.327 \\
\hline 6 & 0.8085 & -8.8440 & 0.0000 & 0.0000 \\
\hline 7 & 0.8021 & -10.2819 & -0.1824 & -8.7200 \\
\hline 8 & 0.8080 & -9.4379 & -0.2400 & 4.8890 \\
\hline 9 & 0.8409 & -11.2784 & -0.0000 & -0.0000 \\
\hline 10 & 0.8363 & -12.5505 & -0.0464 & -1.6000 \\
\hline 11 & 0.8656 & -11.2784 & 0.0000 & 12.8460 \\
\hline 12 & 0.8459 & -11.9463 & -0.0896 & -6.0000 \\
\hline 13 & 0.8568 & -11.9463 & 0.0000 & 8.3606 \\
\hline 14 & 0.8340 & -12.6596 & -0.0496 & -1.2800 \\
\hline 15 & 0.8303 & -12.7331 & -0.0656 & -2.0000 \\
\hline 16 & 0.8357 & -12.4123 & -0.0280 & -1.4400 \\
\hline
\end{tabular}




\begin{tabular}{|l|l|l|l|l|}
\hline 17 & 0.8321 & -12.6800 & -0.0720 & -4.6400 \\
\hline 18 & 0.8227 & -13.2242 & -0.0256 & -0.7200 \\
\hline 19 & 0.8207 & -13.3630 & -0.0760 & -2.7200 \\
\hline 20 & 0.8240 & -13.2058 & -0.0176 & -0.5600 \\
\hline 21 & 0.8264 & -12.9045 & -0.1400 & -8.9600 \\
\hline 22 & 0.8268 & -12.8931 & -0.0000 & 0.0000 \\
\hline 23 & 0.8219 & -13.0453 & -0.0256 & -1.2800 \\
\hline 24 & 0.8175 & -13.1862 & -0.0696 & -5.3600 \\
\hline 25 & 0.8141 & -12.8436 & -0.0000 & 0.0000 \\
\hline 26 & 0.8000 & -13.1792 & -0.0280 & -1.8400 \\
\hline 27 & 0.8188 & -12.4241 & -0.0000 & -0.0000 \\
\hline 28 & 0.8057 & -9.3418 & 0.0000 & 0.0000 \\
\hline 29 & 0.8030 & -13.4075 & -0.0192 & -0.7200 \\
\hline 30 & 0.7938 & -14.1133 & -0.0848 & -1.5200 \\
\hline
\end{tabular}

Power loss in tr. line without UPFC is 0.2 p.u.

WITH UPFC:

When particle swarm optimization algorithm is executed then the following results came in below results the number iteration are shown and the optimal place net of upfc is indicated.

TABLE 2

NUMBER OF ITERATIONS

\begin{tabular}{|c|c|c|}
\hline Iterations & fGBest & fevals \\
\hline 10 & 9935.5 & 330 \\
\hline 20 & 1135 & 630 \\
\hline
\end{tabular}

When UPFC placed between the buses that given by particle swarm algorithm technique,

TABLE 3

BUS VOLTAGE AND POWER FLOW RESULT WITH UPFC

\begin{tabular}{|c|c|c|c|c|}
\hline $\begin{array}{l}\text { Bus } \\
\text { No. }\end{array}$ & $\begin{array}{l}\mathbf{V}_{\text {mag }} \\
(\mathbf{p u})\end{array}$ & $\begin{array}{c}\text { Angle } \\
\text { (degree) }\end{array}$ & $\begin{array}{c}\text { P Flow } \\
\text { (pu) }\end{array}$ & $\begin{array}{c}\text { Q Flow } \\
\text { (pu) }\end{array}$ \\
\hline 1 & 1.0600 & 0.0000 & 0.9451 & 0.9314 \\
\hline 2 & 1.0430 & -5.3585 & 0.9421 & 0.9283 \\
\hline 3 & 1.0189 & -7.5267 & 0.9377 & 0.9239 \\
\hline 4 & 1.0094 & -9.2798 & 0.9360 & 0.9223 \\
\hline 5 & 1.0100 & -14.1846 & 0.9361 & 0.9224 \\
\hline 6 & 1.0086 & -11.0506 & 0.9359 & 0.9221 \\
\hline 7 & 1.0014 & -12.8653 & 0.9346 & 0.9208 \\
\hline 8 & 1.0100 & -11.8277 & 0.9361 & 0.9224 \\
\hline 9 & 1.0366 & -14.0831 & 0.9409 & 0.9271 \\
\hline 10 & 1.0166 & -15.7094 & 0.9373 & 0.9235 \\
\hline 11 & 1.0820 & -14.0831 & 0.9491 & 0.9353 \\
\hline 12 & 1.0466 & -15.1839 & 0.9427 & 0.9289 \\
\hline 13 & 1.0710 & -15.1839 & 0.9471 & 0.9333 \\
\hline 14 & 1.0276 & -16.0515 & 0.9393 & 0.9255 \\
\hline 15 & 1.0194 & -16.0117 & 0.9378 & 0.9240 \\
\hline 16 & 1.0266 & -15.6766 & 0.9391 & 0.9253 \\
\hline 17 & 1.0142 & -15.9128 & 0.9369 & 0.9231 \\
\hline 18 & 1.0000 & -16.4708 & 0.9343 & 0.9206 \\
\hline 19 & 0.9972 & -16.6961 & 0.9338 & 0.9200 \\
\hline 20 & 1.0012 & -16.5100 & 0.9346 & 0.9208 \\
\hline 21 & 1.0028 & -16.2514 & 0.9348 & 0.9210 \\
\hline 22 & 1.0060 & -16.0166 & 0.9354 & 0.9216 \\
\hline 23 & 1.0029 & -16.2595 & 0.9349 & 0.9211 \\
\hline 24 & 0.9917 & -16.3227 & 0.9329 & 0.9191 \\
\hline 25 & 0.9914 & -16.0447 & 0.9328 & 0.9190 \\
\hline 26 & 0.9732 & -16.4871 & 0.9295 & 0.9157 \\
\hline 27 & 1.0000 & -15.5960 & 0.9343 & 0.9206 \\
\hline 28 & 1.0056 & -11.6790 & 0.9354 & 0.9216 \\
\hline 29 & 0.9796 & -16.8850 & 0.9307 & 0.9169 \\
\hline 30 & 0.9679 & -17.8117 & 0.9286 & 0.9148 \\
\hline
\end{tabular}

Power loss in transmission line with UPFC is $0.002116 \mathrm{pu}$.

TABLE 4

OPTIMAL LOCATION OF UPFC

\begin{tabular}{|c|c|c|c|c|}
\hline $\begin{array}{c}\text { Bus } \\
\text { No. }\end{array}$ & $\mathbf{V}_{\text {sh }}(\mathbf{p u})$ & $\begin{array}{c}\text { Theta } \\
\text { (Degree) }\end{array}$ & $\mathbf{P}(\mathbf{p u})$ & $\mathbf{Q}(\mathbf{p u})$ \\
\hline 18 & 1.0000 & -16.4708 & 0.9343 & 0.9206 \\
\hline 27 & 1.0000 & -15.5960 & 0.9343 & 0.9206 \\
\hline
\end{tabular}




\section{Conclusions}

In this paper a PSO based modified method is analyzed for the IEEE 30 bus system by placing a UPFC with Equivalent current injection to achieve optimal location in improving voltage stability of the interconnected transmission system.

The results shown are obtained by performing simulation analysis in MATLAB programming. By inspecting the results, modified Partical Swarm Optimization improves the stability of transmission line by optimal location of UPFC. The UPFC model with ECI is algorithm will provide Power flow calculation with in shortest time and accurate results will be provided.

\section{FUTURE WORK}

The objective of this paper was to find a deterministic approach to the problem of placement of UPFC units with the objective of enhancing the voltage stability margin. In the algorithm developed in this thesis, the optimal location(s) of the UPFC(s) are calculated, but the number of UPFC units is still left to the judgment of optimization problem designer. Certainly there exist situations where the optimal number of UPFC(s) is one, and in other situation the optimal number of units is another. Hence further investigation upon this matter should study a way to integrate the number of UPFC(s) needed in the optimization problem, so that the optimal solution to the new optimization problem has the optimal number of UPFC(s). Further studies can also investigate if there is a optimal configuration of the PSO algorithm to the problems of placement and sizing of not only UPFC unit but of other power network's equipment.

\section{References}

[1] R. Z. Mĩnano, “Optimal power flow with stability constraints,” Ph.D. dissertation, Universidad de Castilla - La Mancha, 2010

[2] V. Ajjarapu, Computational Techniques for Voltage Stability Assessment and Control. Springer, 2006.

[3] P. H. E. Azadani, S. Hosseinian, "Optimal placement of multiple statcom for voltage stability margin enhancement using particle swarm optimization," Springer Berlin Electrical Engineering (Archiv fur Elektrotechnik), pp. 503-510, 2008.

[4] Y. del Valle, J. C. Hernandez, G. K. Venayagamoorthy, and R. G. Harley, "Multiple statcomallocation and sizing using particle swarm optimization," Power Systems Conference and Exposition, pp. 1884-1891, 2006.

[5] P. Kundur, Power System Stability and Control.McGraw-Hill, 1993.

[6] J. P. S. Paiva, Redes de Energia El 'ectrica - Uma An' aliseSist 'emica, 2nd ed. IST Press, 2007.

[7] M. B. Keskin, "Continuation power flow and voltage stability in power systems," Master's thesis, The Graduate School of Natural and Applied Sciences of Middle East Technical University, September 2007.

[8] C. A. Ca nizares, "Voltage collapse and transient energy function analyses of ac/dc systems," Ph.D. dissertation, University of Wisconsin-Madison, 1991.

[9] F. Milano, Power System Modelling and Scripting. Springer, 2010.

[10] W. F. Tinney and C. E. Hart, "Power flow solution by newton's method," IEEE Transactions on Power Apparatus and Systems, pp. 1449-1460, 1967.

[11] V. Ajjarapu and C. Christy, "The continuation power flow: A tool for steady state voltage stability analysis," IEEE Transactions on Power Systems, pp. 416-423, 1992.

[12] J. Jasni, S. Bahari, N. Mariun, M. Z. A. Kadir, and H. Hizam, "State of the art for voltage collapse point approximation using continuation power flow,” European Journal of Scientific Research, pp. 98-105, 2008. 